\title{
Calibration of fatigue performance model for cement stabilized flexible pavements
}

\author{
E. Rodriguez \& R. Ashtiani \\ Department of Civil Engineering, The University of Texas at El Paso, Texas, USA
}

\begin{abstract}
Chemical stabilization of reclaimed and marginal materials with cement has proven to be a viable option for sustainable design and construction of flexible pavements. The primary role of the treated base layers is to provide a robust platform that withstands complex stress paths imparted by moving traffic loads. In addition to considerations of loadrelated distresses in the structural design process of pavements with cement treated layers, the selection of appropriate cement content in the laboratory mixture design greatly influences the service life of pavements. For instance, excessive cement content in base mixes can potentially result in shrinkage cracking that will further propagate to the surface layers. The primary focus of this study was to develop and calibrate a new generation of fatigue performance model with considerations of the traffic-induced stresses, as well as the shrinkage parameters in the laboratory mixture design of cement treated layers. To achieve this objective, a comprehensive laboratory experiment design was developed to characterize the tensile and shrinkage behavior of cement stabilized virgin and reclaimed materials. Subsequently, a logical performance model was proposed and further calibrated using 51 pavement sections. Ultimately, the performance of the developed model was tested on a new set of data for crossvalidation purposes. The proposed calibrated fatigue performance model with the inclusion of IDT and shrinkage cracking characteristics of the cement treated materials provides a practical approach for the design of pavements with cement stabilized foundations.
\end{abstract}

Keywords: Cement stabilization, Recycled concrete aggregate, Full depth reclamation, Fatigue performance model, Model Calibration

\section{INTRODUCTION}

The application of calcium-based treatment agents such as cement, lime, fly ash, among others, is a sustainable means to improve the mechanical properties of pavement foundations with reclaimed materials. Improved orthogonal load-bearing capacity and enhanced durability of cement treated layers are the primary motivations for pavement engineers to include stabilization strategies in their toolbox for the design of new roads and the rehabilitation of existing pavement structures. The use of an adequate amount of cement during the design of cement stabilized mixes will considerably influence the structural and functional performance of a pavement structure. For example, stabilized granular base mixes with excessive amounts of cement are prone to develop shrinkage cracking that will propagate to the surface layers and ultimately jeopardize the longevity of the pavement structure.

Even though the mechanical properties of cement treated materials have been well studied, very limited research has been conducted to relate such properties to the performance of pavement structures (Mohammad et al., 2000, Saxena et al., 2010, Molenaar \& Pu, 2008; Wu 
et al., 2011; Li et al., 2019). The latest pavement design methodologies, such as The Mechanistic-Empirical Pavement Design Guide or MEPDG (ARA \& NCHRP, 2004) and the Texas Mechanistic-Empirical Flexible Pavement Design System or TxME (Hu et al., 2014), are based on performance models to predict fatigue cracking and permanent deformation of pavement structures with cement stabilized layers. Unfortunately, the fatigue performance model incorporated in current versions of the MEPDG and the TxME for cement treated layers have proven to be impractical and not yet calibrated.

To overcome this issue, several researchers have developed and calibrated new performance models applicable to cement stabilized materials. Wu et al. (2011) developed a finite element model to simulate the permanent deformation behavior of cement treated materials in flexible pavements. The proposed model was calibrated using surface permanent deformation data from six accelerated pavement test sections constructed in Louisiana. The authors reported good agreement between the predicted and measured rut depths in two low-volume roads. In a relevant study, Li et al. (2019) developed and calibrated a bottom-up and top-down fatigue model for cement stabilized layers in flexible pavements, with considerations of variations of modulus due to erosion, as well as freeze-thaw and wet-dry cycles. The researchers calibrated the performance model based on field-measured modulus values obtained from Falling Weight Deflectometer (FWD) backcalculated data. Saxena et al. (2010) provided a discussion on the shortcomings of the characterization method and performance models in the MEPDG. The authors postulated that the MEPDG fatigue model does not adequately address the effect of shrinkage cracking. Therefore, they recommended the incorporation of a shrinkage distress model for comprehensive modeling of cement stabilized materials in the MEPDG.

The current version of the material models incorporated in the MEPDG overlooks the significance of shrinkage cracking. The primary criterion in the current model is the ratio of the loadinduced tensile stresses and modulus of rupture of materials. Ashtiani and Tarin (2016) outlined the theoretical and practical issues regarding the flexural bending beam test for lightly and heavily cement treated base layers. The researchers performed a series of numerical simulations of the test considering different material properties and stress paths. Furthermore, the authors evaluated the mechanical behavior of 570 specimens as part of the laboratory experiment design. Through extensive numerical modeling complemented by laboratory testing, the authors showed in some permutations, that nearly $35 \%$ of the cross-section of the beams stayed in compression in unique bending loading conditions. Therefore, the traditional equations with assumptions of linear distribution of the tensile stresses along the transverse direction of the beam greatly overestimate the tensile capacity of cement treated base materials. In addition to theoretical concerns, the authors outlined practical issues, such as non-uniformity of compaction in $15.24 \times 15.24 \times 50.8 \mathrm{~cm}$ prismatic beams, and disintegration of large beams during demolding, handling, and transportation to the test set up for lightly cement stabilized base and subgrade soils. Therefore, the authors provided a modification to the indirect diametrical tensile (IDT) test for an efficient and repeatable alternative for determining the tensile strength properties of cement treated base materials in the laboratory.

The primary objective pursued in this study was to develop and calibrate a novel performance model for cement stabilized base layers. The model incorporates IDT strength, as well as both load-induced and shrinkage cracking behavior of cement treated foundations. To accomplish this objective, a comprehensive laboratory experiment design coupled with numerical simulations were developed to characterize the tensile and shrinkage behavior of cement stabilized virgin and reclaimed materials. Subsequently, a logical performance model was proposed, calibrated, and further validated using 64 pavement sections lasting in service for 85 years, on average. $80 \%$ of pavement sections (51 sections) were used to calibrate the model while the remaining 20\% (13 sections) were used for cross-validation purposes. 


\section{METHODOLOGY}

Figure 1 shows the methodology for the development and calibration of the predictive model for the estimation of the fatigue performance of cement stabilized virgin and reclaimed base materials in flexible pavement structures. The process includes three major areas that will be discussed in detail in the upcoming sections: Development of Fatigue Performance Model, Field Data Collection and Analysis, and Field Calibration and Validation Algorithm. Relevant databases were collected based on laboratory test results, finite element simulations, and field testing for each subcategory illustrated in Figure 1.

In this study, a total of 120 specimens, considering replicates were fabricated and subjected to various laboratory tests. The Unconfined Compressive Strength (UCS) test, static and dynamic IDT strength tests, submaximal modulus tests, and the coefficient of thermal expansion (COTE) or shrinkage test were incorporated in the laboratory experimental design to characterize compressive and tensile strengths, shrinkage behavior, resilient properties, and permanent deformation potential of cement stabilized materials. Four different aggregate base materials including crushed limestone and siliceous gravel, as virgin aggregates, and two sources of reclaimed materials including Recycled Concrete Aggregate (RCA) and blends of Full Depth Reclamation (FDR) were integrated in the experiment design. Three levels of cement contents, $2 \%, 3 \%$, and $4 \%$, were added to the mixtures to evaluate the contribution of the calcium-based treatment agents in lightly stabilized and heavily stabilized permutations.

Finally, a total of 64 pavement sections were used in the calibration and cross-validation process of the newly developed fatigue performance model for cement treated base layers.

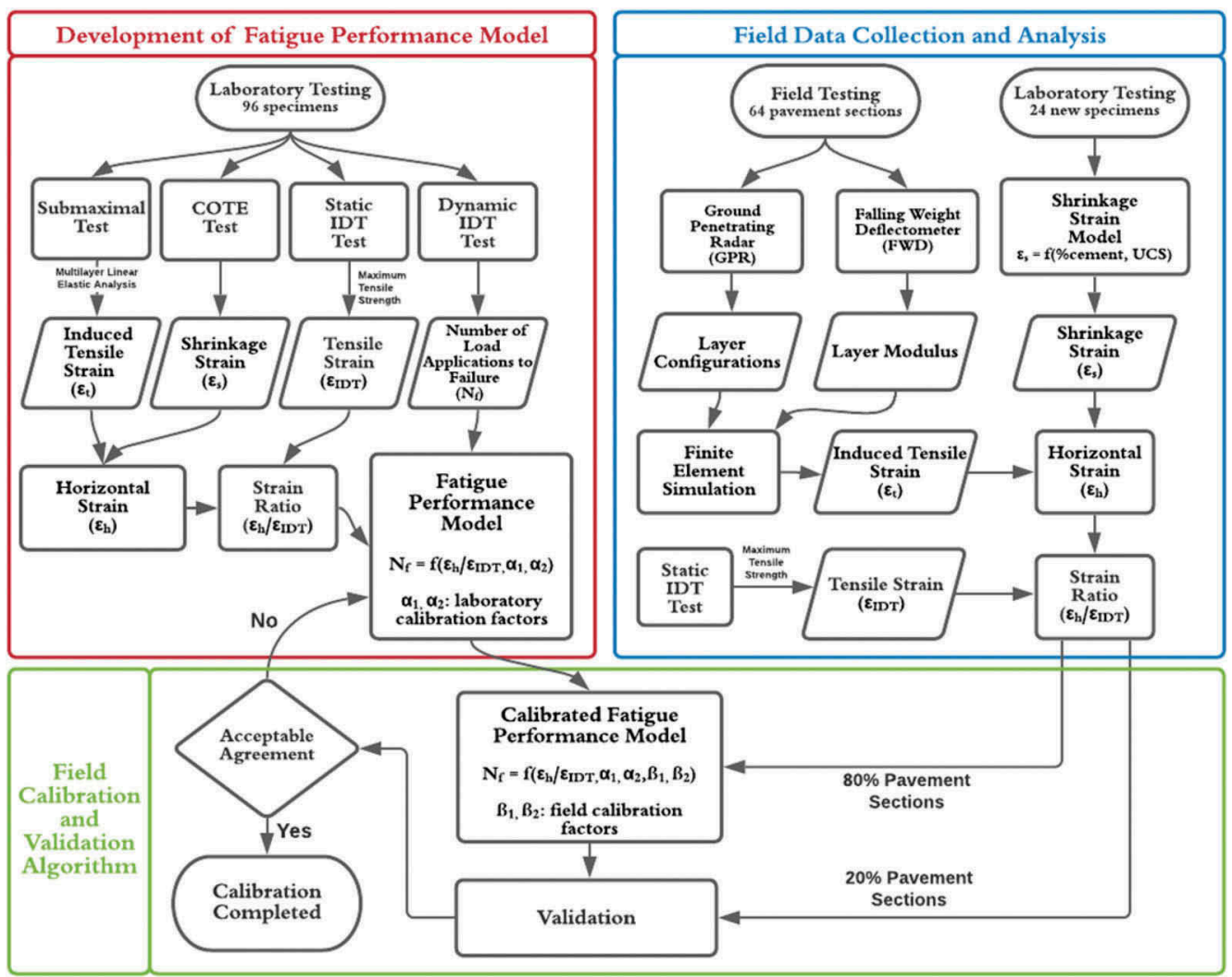

Figure 1. Development, calibration, and validation process of the fatigue performance model. 


\section{DEVELOPMENT OF FATIGUE PERFORMANCE MODEL}

The fatigue performance model developed in this study is based on the strain-ratio concept for the prediction of the fatigue life of cement treated materials. In establishing the fatigue performance model, traffic-induced strain, shrinkage strain, and failure strain in the IDT test were incorporated in the model to provide a holistic view of field conditions. The strain ratio $\left(\varepsilon_{h} / \varepsilon_{I D T}\right)$ was computed based on strain values equivalent to the horizontal strain $\left(\varepsilon_{h}\right)$, as the summation of tensile strain induced by traffic loads $\left(\varepsilon_{t}\right)$ from finite element simulations and shrinkage strain from laboratory tests, as well as the strain that corresponds to the maximum tensile strength of materials $\left(\varepsilon_{I D T}\right)$.

Strain values that corresponded to the $\varepsilon_{I D T}$ and shrinkage strain $\left(\varepsilon_{s}\right)$ were obtained in the laboratory from the static IDT test and shrinkage test, respectively. Multilayer linear elastic analysis was conducted to compute $\varepsilon_{t}$ at the bottom of the cement treated layer for sitespecific traffic conditions, pavement layer configurations, layer thicknesses, and modulus values. The moduli for cement treated layers were obtained experimentally after conducting the submaximal modulus test on cement stabilized virgin and reclaimed materials in the laboratory.

The selected form of the fatigue performance model was based on the relationship between the strain ratio and the number of load applications to failure $\left(N_{f}\right)$. The latter parameter was obtained experimentally from the dynamic IDT test. The rationale to relate $\varepsilon_{h} / \varepsilon_{I D T}$ to $N_{f}$ was to take into consideration the notable effect of shrinkage cracking on the bottom-up tensilefatigue life of cement stabilized base layers. Figure 2 provides the relationship between $N_{f}$ and the strain ratio for cement treated virgin aggregates and cement treated reclaimed materials.

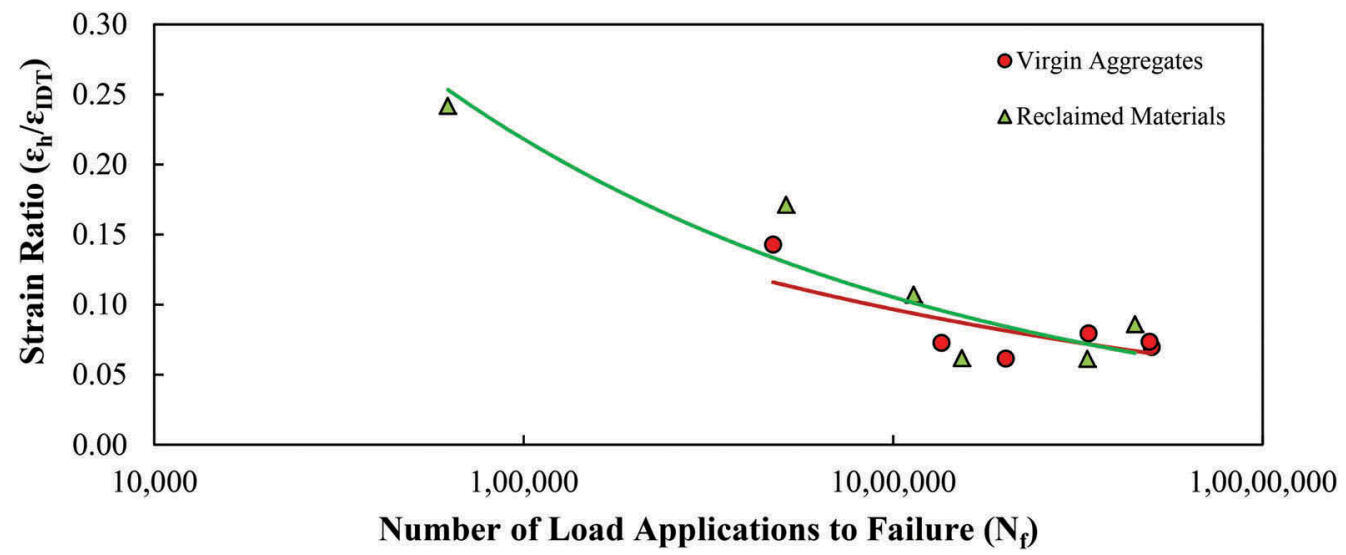

Figure 2. Relationship between the number of load applications to failure $\left(N_{f}\right)$ and strain ratio $\left(\varepsilon_{h} / \varepsilon_{I D T}\right)$ for cement stabilized virgin aggregates and reclaimed materials.

As depicted in Figure 2, the descending nature of the trendlines suggests that permutations with higher shrinkage strains and taxing loading scenarios manifested by higher tensile strains exhibit lower fatigue life compared to other counterparts. Furthermore, the slope of the best fit lines in the fatigue failure and strain ratio plots are appreciably different for the two groups of materials tested in this study. This underscores the influence of the type of virgin and reclaimed material to partake in hydration and strength reactions, thus resulting in mixtures with different fatigue performances. Equation 1 shows the general form of the fatigue performance model for cement stabilized materials in this study. 


$$
\ln \left(N_{f}\right)=\frac{\beta_{1} x \alpha_{1}-\left(\varepsilon_{h} / \varepsilon_{I D T}\right)}{\beta_{2} x \alpha_{2}}
$$

Where: $N_{f}$ is the number of load repetitions to failure, $\varepsilon_{h} / \varepsilon_{I D T}$ is the strain ratio, $\alpha_{1}$ and $\alpha_{2}$ are laboratory calibration factors, and $\beta_{1}$ and $\beta_{2}$ are field calibration factors.

The dynamic IDT results were further used for the determination of two sets of laboratory shift factors, $\alpha_{1}$ and $\alpha_{2}$ for virgin and reclaimed materials. The authors believe it is prudent to separate the databases for virgin and reclaimed materials as their mechanical behavior was observed to be drastically different in the laboratory. Based on the curve fitting procedure adopted in this study, the laboratory shift factors for virgin aggregates, $\alpha_{1}$ and $\alpha_{2}$, were 0.4561 and 0.026 , respectively. For reclaimed materials, the model parameters $\alpha_{1}$ and $\alpha_{2}$, were 0.7163 and 0.043 , respectively. Section 5 of this paper provides the procedure for the estimation of the field calibration parameters, $\beta_{1}$ and $\beta_{2}$, values.

\section{FIELD DATA COLLECTION AND ANALYSIS}

\subsection{Field data collection}

A comprehensive database of cross-sectional characteristics and material properties using nondestructive testing equipment, such as FWD and ground penetrating radar (GPR), was compiled from 64 pavement sections. The evaluated pavement sections covered a broad range of site characteristics. The authors combined the field database with available databases from published relevant literature. The combined dataset was incorporated into the calibration algorithm and cross-validation process of the fatigue performance model. The database required for executing the calibration and validation of the model included the location of the pavement sections, layers thickness (based on design blueprints and GPR results), backcalculated FWD modulus, materials properties of stabilized base layers (i.e., UCS, maximum dry density, optimum moisture content), and type of material for each layer.

Upon finalizing the pavement feature database, the next step corresponded to the determination of the traffic-induced tensile strains using finite element simulations.

\subsection{Pavement response calculations by Finite Element (FE) method}

To simulate field conditions and calculate critical pavement responses, the authors used the FE software Abaqus to model the 64 pavement sections. Different layer configurations of pavement structures were simulated in the FE program. Figure 3 (a) exhibits an example of meshing, boundary conditions, geometry of layers, and traffic-induced tensile strain results for a representative pavement section that contains an asphalt concrete surface layer (AC), cement treated base layer (CTB), and the subgrade soil (SG).

Site-specific structural properties of pavements were assigned to each representative field roadway section in the FE model. Since the entire three-dimensional model was symmetric, only a quarter size of the structure was simulated. This led to optimizing the computational efficiency of the analysis. A finer mesh was defined under the wheelpath, while a coarser one was used in regions far from the loading area. Bottom and lateral boundaries of the FE model were appropriately defined to assure a realistic field model.

The traffic loads considered for the simulation of field roadway sections were based on the historic traffic (ranging from 4.8 million to 28.5 million equivalent single axle loads), as well as the most frequent truck classes and tire pressure in the studied network (Ashtiani et al., 2019). FE models were developed based on tandem wheel loads with 42.7 $\mathrm{kN}$, a contact area of $274.2 \mathrm{~cm}^{2}$, a tire pressure of $779.1 \mathrm{kPa}$, and a wheel spacing of $30.48 \mathrm{~cm}$. Lastly, pavement structures associated with distinct materials properties and layer configurations were analyzed for specific sites to obtain the traffic-induced tensile strains $\left(\varepsilon_{t}\right)$ as displayed in Figure $3(\mathrm{~b})$. 
Upon finalizing the estimation of $\varepsilon_{t}$ at the bottom of the cement stabilized base layer in all pavement sections, the upcoming step was related to the determination of $\varepsilon_{s}$ and $\varepsilon_{I D T}$. These strain values are mainly relevant to calculate $\varepsilon_{h}$ and, consequently, calculate $\varepsilon_{h} / \varepsilon_{I D T}$ of the evaluated field sections.

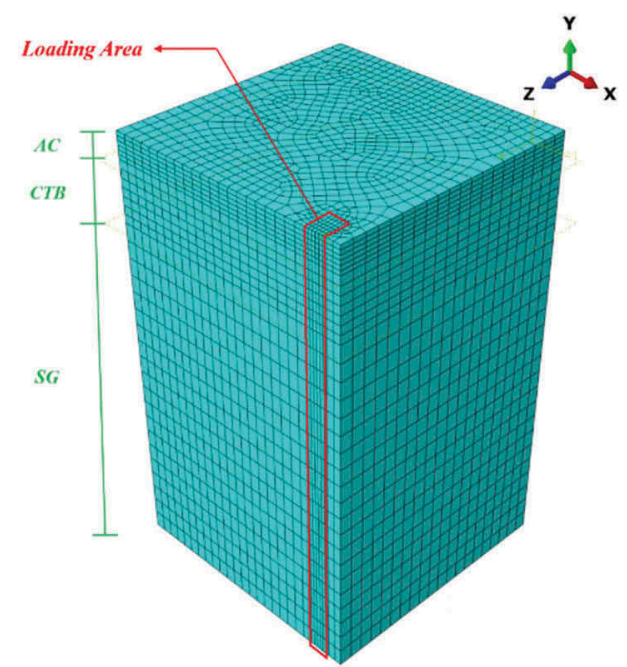

(a)
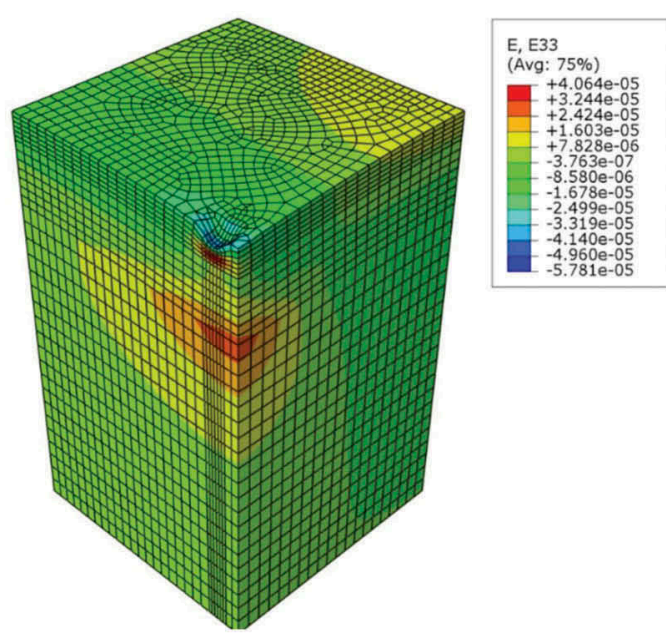

(b)

Figure 3. (a) Meshing and pavement layers simulation in Abaqus. (b) Pavement responses contours for induced tensile strain $\left(\varepsilon_{t}\right)$.

\subsection{Determination of the shrinkage strain the laboratory}

Shrinkage strain $\left(\varepsilon_{s}\right)$ values for the stabilized base layers of pavement sections in the field were calculated based on a shrinkage model developed in the laboratory suitable for cement treated virgin aggregates and reclaimed materials (Ashtiani et al., 2020). A total of 48 treated specimens with 2, 3, and 4\% of cement were prepared and subjected to UCS and COTE tests. Four different aggregate sources including crushed limestone and siliceous gravel, as virgin aggregates, and RCA and FDR, as reclaimed materials, were incorporated in the experiment design to develop the shrinkage model. Multi-variate regression analysis was used to establish robust relationships between shrinkage strain, cement content, and unconfined compressive strength. Equation 2 shows the model for the prediction of shrinkage strain in cement stabilized systems.

$$
\varepsilon_{s}=0.000187+1076.69 \times \frac{C \%^{2}}{S_{C}}
$$

Where: $\varepsilon_{s}$ is the shrinkage strain, $C \%$ is the percent of cement content, and $S_{C}$ is the unconfined compressive strength $(\mathrm{kPa})$.

The prediction model developed by Ashtiani et al. (2020) provided a reasonably good estimate of the shrinkage strain of cement stabilized base materials. Statistical results showed good agreement between the predicted and laboratory-measured shrinkage strain after seven days of temperature cycles.

Upon completing the calculation of shrinkage strain $\left(\varepsilon_{s}\right)$ of field roadway sections, the horizontal strain $\left(\varepsilon_{h}\right)$ was determined based on the summation of the induced tensile strain $\left(\varepsilon_{t}\right)$ and the shrinkage strain $\left(\varepsilon_{s}\right)$ to consider the impact of shrinkage behavior on 
the cement stabilized base layers. The further analysis consisted of calculating the strain ratios of the cement treated base layers from field pavement sections. Therefore, it deemed necessary to determine the strains that corresponded to the maximum tensile strength of materials $\left(\varepsilon_{I D T}\right)$.

Figure 4 Shows the relationship between the maximum tensile strength and its corresponding strain obtained for different types of materials with different stabilizer percentages. As evidenced in Figure 4, there is a narrow range for the variation of shrinkage strain for different types of materials.

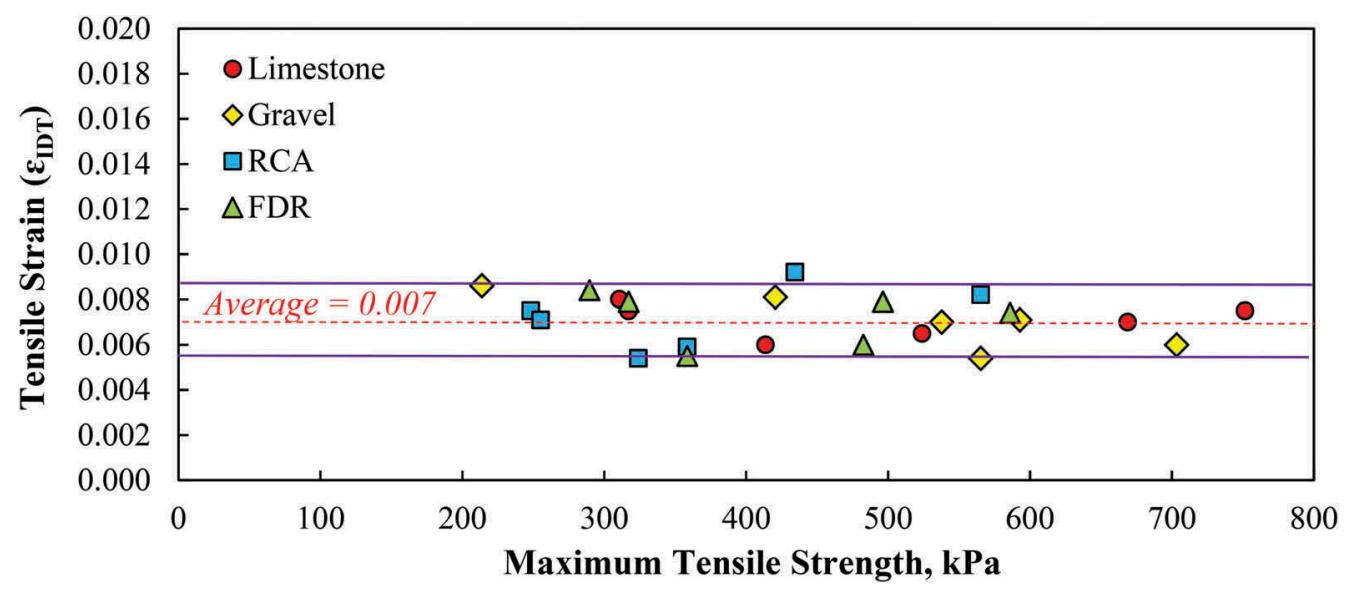

Figure 4. Relationship between tensile strain $\left(\varepsilon_{I D T}\right)$ and maximum tensile strength.

For each cement stabilized material considered in this study, the authors calculated the coefficient of variation (CV) and range of $\varepsilon_{I D T}$ as illustrated in Figure 5. As observed in the plot, the average strain values corresponding to the maximum tensile strength for virgin aggregates and reclaimed materials were 0.0070 and 0.0072 , respectively. The statistical analysis showed that $\varepsilon_{I D T}$ had a coefficient of variation lower than $20 \%$ in all cases. This implies that the scatter of the data points revolves around the average value.

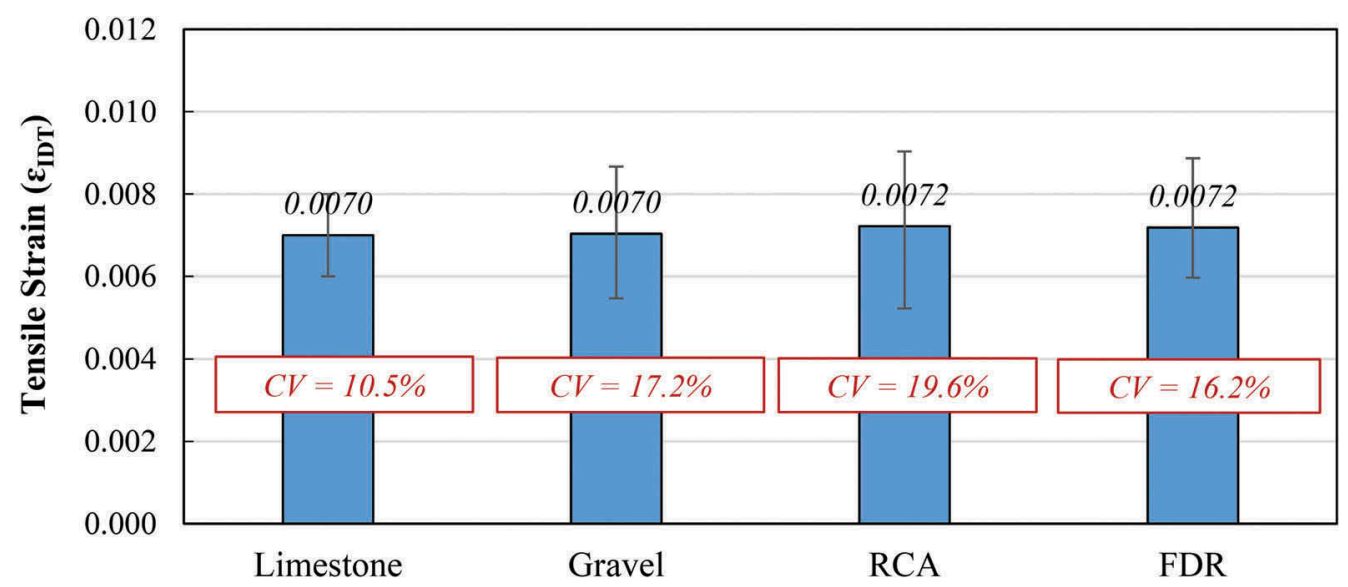

Figure 5. Tensile strain corresponding to the maximum tensile strength $\left(\varepsilon_{I D T}\right)$ for different cement stabilized materials. 


\section{FIELD CALIBRATION PARAMETERS AND VALIDATION ALGORITHM}

To provide credibility to the structural design process of pavements with cement treated base layers, the study incorporated a field calibration process for the novel fatigue performance model. The split-sample approach was used to verify the accuracy of the prediction model during this calibration effort. Approximately $80 \%$ of the sections (51 field pavement sections) were randomly selected for field calibration purposes and $20 \%$ of the sections (13 field pavement sections) were selected for cross-validation.

Laboratory calibration factors (i.e., $\alpha_{1}$ and $\alpha_{2}$ ) of the fatigue performance model were adjusted to improve precision using the representative field database consisted of 51 pavement sections. The predicted strain ratios in the laboratory were compared to the strain ratios obtained from pavement sections based on the collected field database for stabilized virgin aggregates and stabilized reclaimed materials. More details on the parameters included in the database are provided in Ashtiani et al. (2020). Figure 6 presents the initial relationship between predicted and measured strain ratios before calibration.

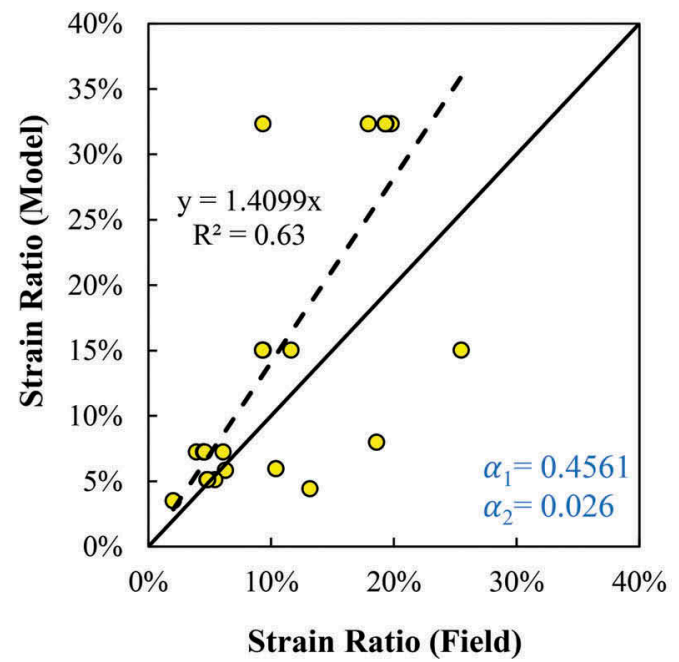

(a)

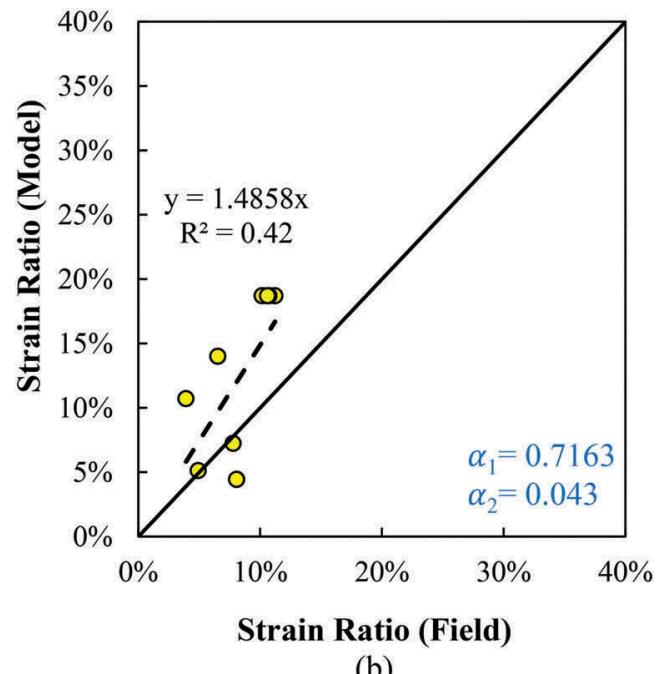

(b)

Figure 6. Predicted (model) versus measured (field) strain ratios before calibration for (a) stabilized virgin base layers and (b) stabilized reclaimed base layers.

Field calibration factors (i.e., $\beta_{1}$ and $\beta_{2}$ ) were introduced in the fatigue performance model to eliminate the bias and reduce the standard error between the predicted and the measured strain ratios. Figure 7 shows the final relationship between predicted and measured strain ratios considering calibration factors. As observed in the plot, bias was eliminated and the precision was improved for the developed fatigue performance model. Based on the comparison of strain ratios, $83 \%$ of predicted strain ratios had a difference lower than $5 \%$ when compared to the measured strain ratios for stabilized virgin base layers. Similarly, $80 \%$ of predicted strain ratios had a difference lower than $5 \%$ when compared to the measured strain ratios for stabilized reclaimed base layers.

Laboratory and field calibration factors of the model for different cement stabilized materials are tabulated in Table 1. The results clearly show improvements in the predicted values after incorporation of the field calibration values. 
Table 1. Laboratory and field calibration factors of the fatigue performance model.

\begin{tabular}{lllllll}
\hline Fatigue Performance Model & $\alpha_{1}$ & $\alpha_{2}$ & $\beta_{1}$ & $\beta_{2}$ & $\mathrm{R}$ & $\mathrm{R}^{2}$ \\
\hline Model for Virgin Aggregates & 0.4561 & 0.026 & 1.40 & 1.36 & 0.85 & 0.73 \\
Model for Reclaimed Materials & 0.7163 & 0.043 & 1.16 & 1.09 & 0.81 & 0.65 \\
\hline
\end{tabular}

As stated earlier, the field calibrated model was cross-validated using an independent set of data $(20 \%$ of the field dataset) to evaluate the predictions. The validation results are shown in Figure 7. The close scatter of the validation data cloud around the equality line is an indication of the capability of the model to provide a reasonable agreement between the prediction and field conditions. Additionally, the maximum differences between predicted and measured strain ratios were $6.7 \%$ and $5.0 \%$ for virgin and reclaimed materials, respectively.

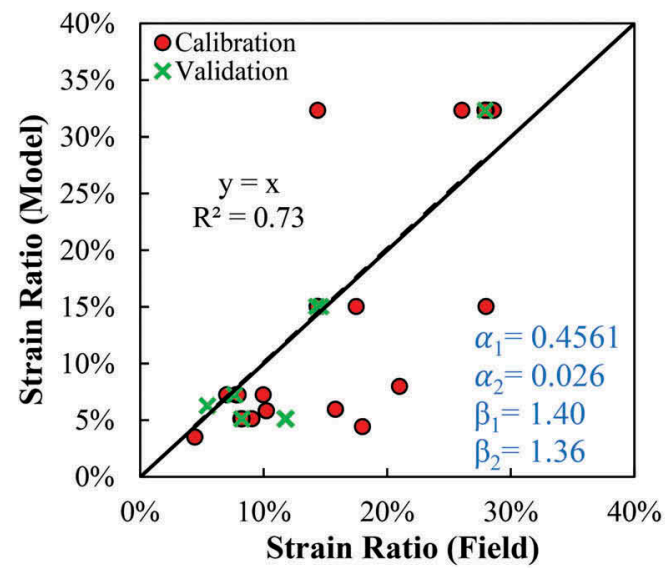

(a)

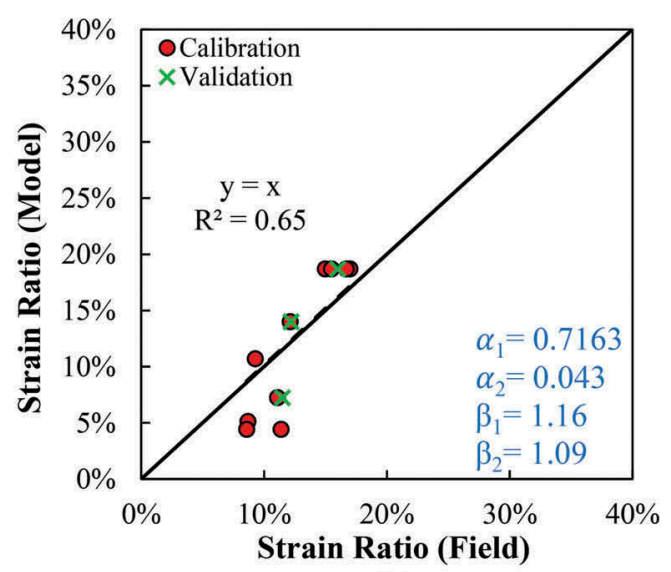

(b)

Figure 7. Predicted (model) versus measured (field) strain ratios after calibration for (a) stabilized virgin base layers and (b) stabilized reclaimed base layers.

\section{CONCLUSIONS}

The focus of this research effort was to develop a new fatigue performance model for cement treated virgin and reclaimed base materials incorporating load-induced, as well as shrinkage cracking behavior of cement stabilized base layers. To achieve this objective, multiple tests including the UCS test, static and dynamic IDT tests, submaximal modulus tests, and shrinkage tests were incorporated in the laboratory experiment design to characterize compressive and tensile strengths, shrinkage behavior, resilient properties, and permanent deformation potential of cement stabilized materials. A total of 120 treated specimens were prepared with four different granular material sources, namely crushed limestone and siliceous gravel, as virgin aggregates, and RCA and FDR materials, as reclaimed aggregates. All four aggregate sources were stabilized with $2 \%, 3 \%$, and $4 \%$ cement. Therefore, the model incorporates both lightly stabilized systems, as well as heavily stabilized aggregate matrices. The primary divergence from existing fatigue performance models in this study was the departure from using modulus of rupture and incorporation of the IDT test and the shrinkage strain for the estimation of fatigue life of pavements with cement treated base layers.

The study also aimed to calibrate and cross-validate the proposed fatigue performance model based on field performance data. This objective was achieved by developing a comprehensive catalog of cross-sectional features and material properties of 64 
pavement sections based on non-destructive field testing, such as FWD and GPR, in combination with available databases from published pertinent literature. The fatigue performance model was calibrated for 51 pavement sections with different cross-sectional characteristics and material properties to enhance the generalization of the model. The precision of the fatigue performance model was evaluated on a new set of 13 pavement sections for cross-validation purposes. The statistical results show the superiority of the newly developed model to better represent the fatigue performance of pavements with cement stabilized base layers.

\section{REFERENCES}

Applied Research Associates, and Inc. National Cooperative Highway Research Program, 2004. Guide for Mechanistic-Empirical Design for New and Rehabilitated Pavement Structures. Final Report, NCHRP 1-37A. Transportation Research Board, National Research Council.

Ashtiani, R.S. and Tarin, J., 2016. Testing Procedures for Long Life Heavy Duty Stabilized Bases. Report No. FHWA/TX-06/0-5223-2, The University of Texas at El Paso's Center for Transportation Infrastructure Systems, Austin, Texas.

Ashtiani, R.S., Morovatdar, A., Licon, C., Tirado, C., Gonzales, J., and Rocha, S., 2019. Characterization and Quantification of Traffic Load Spectra in Texas Overweight Corridors and Energy Sector Zones. Report No. FHWA/TX-19/0-6965-1, The University of Texas at El Paso's Center for Transportation Infrastructure Systems, Austin, Texas.

Ashtiani, R.S., Rashidi, M., Rodriguez, E., Ordaz, M., Cruz H., Garay, G., and Rocha, S., 2020. Establishing Best Practices for Construction and Design of Cement Treated Materials. Report No. FHWA/ TX-19/0-6949-0, The University of Texas at El Paso's Center for Transportation Infrastructure Systems, Austin, Texas.

Hu, S., Zhou, F., and Scullion, T., 2014. Development of Texas Mechanistic-Empirical Flexible Pavement Design System (TxME). Report No. FHWA/TX-14/0-6622-2, Performed in cooperation with the Texas Department of Transportation and the Federal Highway Administration, College Station, Texas.

Li, X., Wang, J., Wen, H., and Muhunthan, B., 2019. Field Calibration of Fatigue Models of Cementitiously Stabilized Pavement Materials for Use in the Mechanistic-Empirical Pavement Design Guide. Transportation Research Record, No. 2673, pp. 427-435.

Mohammad, L.N., Raghavandra, A., and Huang, B., 2000. Laboratory Performance Evaluation of Cement-Stabilized Soil Base Mixtures. Transportation Research Record, No. 1721, pp. 19-28.

Molenaar, A.A.A., and Pu, B., 2008. Prediction of Fatigue Cracking in Cement Treated Base Courses. Pavement Cracking: Mechanisms, Modeling, Detection, Testing, and Case Histories, Taylor \& Francis Group.

Saxena, P., Tompkins, D., Khazanovich, L., and Balbo, J.T., 2010. Evaluation of Characterization and Performance Modeling of Cementitiously Stabilized Layers in the Mechanistic-Empirical Pavement Design Guide. Transportation Research Record, No. 2186, pp. 111-119.

Wu, Z., Chen, X., Yang, X., and Zhang, Z., 2011. Finite Element Model for Rutting Prediction of Flexible Pavement with Cementitiously Stabilized Base-Subbase. Transportation Research Record, No. 2226, pp. 104-110. 